\title{
Ueber die Formen der Curven dritter Ordnung.
}

\author{
(Von Herrn H. Durège in Prag.)
}

Schon frühe haben die Geometer es sich angelegen sein lassen, die Curven dritter Ordnung nach gewissen Eigenschaften zu classificiren, um über die mannigfaltigen Gestalten derselben einen Ueberblick zu gewinnen. Solche Classificationen gaben Newton in der Enumeratio linearum tertii ordinis, 1711; Stirling in der Schrift: Lineae tertii ordinis Newtonianae, sive illustratio tractatus Newtoni de enumeratione linearum tertii ordinis, 1717; Euler in dem zweiten Bande der Introductio in analysin infinitorum, 1748; Plïcker in dem System der analytischen Geometrie, 1835. Diese Classificationen gewähren aber keine vollständige Befriedigung, da sie wesentlich auf einer analytischen Grundlage ruhen, ihre Eintheilungsgründe nicht oder doch nicht ausschliesslich von rein geometrischen Eigenschaften hernehmen und deswegen nicht einen klaren Ueberblick über die Gestaltungen dieser Linien gestatten. Einen erheblichen Fortschritt machte Möbius in der Abhandlung über die Grundformen der Linien der dritten Ordnung. *) Er hob darin die wesentlichen Unterschiede vor den unwesentlicheren klar hervor und vereinfachte dadurch die Classification um ein Beträchtliches. Seine Eintheilung lässt jedoch in einem Punkte noch etwas zu wünschen übrig, nämlich darin, dass sie nicht erkennen lässt, was die verschiedenen Curvenformen in ihren geometrischen Eigenschaften Gemeinsames oder Verschiedenes darbieten. Zu dieser Erkenntniss kann man aber gelangen, wenn man die Beschaffenheit der vier von einem Punkte der Curve an die letztere gehenden Tangenten näher betrachtet.

I.

Wir sondern von den übrigen Curven diejenigen $a b$, welche einen Doppel- oder Rückkehrpunkt besitzen, weil diese Specialitäten bilden, und beschäftigen uns zunächst nur mit den allgemeinen Curven dritter Ordnung, von deren Punkten immer vier (reelle oder imaginäre) Tangenten ausgehen. Zur Erkenntniss der Beschaffenheit dieser Tangenten führen folgende Sätze:

*) Abhandlungen der k. sächs. Gesellschaft der Wissenschaften, Bd. 1. 1849. 
1. Wenn aus einem Punkte $\alpha$ einer Curve dritter Ordnung vier reelle Tangenten mit den Berührungspunkten $a_{1} a_{2} a_{3} a_{4}$ an die Curve gelegt werden können, und es lässt sich aus einem anderen Curvenpunkte $\beta$ eine reelle Tangente $\beta b_{1}$ an die Curve legen, so gehen von $\beta$ allemal vier reelle Tangenten an die Curve.

Beweis. Schneidet man die Curve mit $b_{1} a_{1}$ in $\gamma$ und zieht aus $\gamma$ die Strahlen $\gamma\left(a_{1} a_{2} a_{3} a_{4}\right)$, so sind die Punkte $b_{1} b_{2} b_{3} b_{4}$, in welchen diese Strahlen die Curve treffen, die Berührungspunkte der von $\beta$ ausgehenden Tangenten. ${ }^{*}$ ) Diese aber sind alle reell, wenn $a_{1} a_{2} a_{3} a_{4}$ und $b_{1}$ es sind.

2. Wenn die aus einem reellen Curvenpunkte $\beta$ an die Curve gehenden Tangenten $\beta\left(b_{1} b_{2} b_{3} b_{4}\right)$ alle vier imaginär sind, und es lässt sich aus einem anderen reellen Curvenpunkte $\alpha$ eine reelle Tangente $\alpha a_{1}$ an die Curve legen, so sind alle vier von $\alpha$ ausgehenden Tangenten $\alpha\left(a_{1} a_{2} a_{3} a_{7}\right)$ reell.

Beweis. Man schneide die Curve mit $\alpha \beta$ in $\gamma$ und ziehe auch die aus $\gamma$ an die Curve gehenden Tangenten $\gamma\left(c_{1} c_{2} c_{3} c_{4}\right)$ mit in die Betrachtung hinein. Dann liegen die 12 Berührungspunkte $a_{1} a_{2} a_{3} a_{4}, b_{1} b_{2} b_{3} b_{4}, c_{1} c_{2} c_{3} c_{4}$ zu je drei auf 16 Geraden und können in Beziehung auf diese Eigenschaft in folgender Art gruppirt werden: ${ }^{* *}$ )

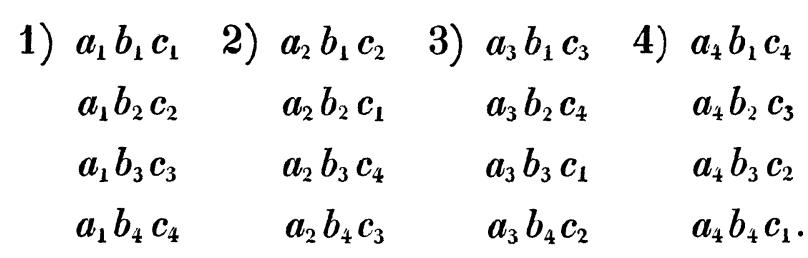

Da nun die vier der Annahme nach imaginären Tangenien $\beta\left(b_{1} b_{2} b_{3} b_{4}\right)$ von einem reellen Curvenpunkte ausgehen, so sind sie, so wie auch ihre Berührungspunkte paarweise conjugirt imaginär. Seien $b_{1} b_{2}$ und $b_{3} b_{4}$ diese Paare. Dann sind in der ersten Gruppe die Geraden $a_{1} b_{1} c_{1}$ und $a_{1} b_{2} c_{2}$ imaginär, da keine von ihnen durch zwei conjugirt imaginäre Punkte geht; sie sind aber conjugirt imaginär, da sie sich in einem reellen Punkte $a_{1}$ schneiden, und jede von ihnen durch einen der conjugirt imaginären Punkte $b_{1}$ und $b_{2}$ geht; mithin sind auch $c_{1}$ und $c_{2}$ conjugirt imaginäre Punkte. Dasselbe gilt von $c_{3}$ und $c_{4}$. Nun muss unter den von $\alpha$ ausgehenden Tangenten ausser $\alpha a_{1}$ mindestens noch eine reell sein. Sei diese $\alpha a_{2}$, dann liefert die zweite Gruppe nichts

*) Vgl. des Verfassers „Curven dritter Ordnung,“ art. 382.

**) Curven dritter Ordnung, art. 385 . 
Neues, sondern bestätigt nur das vorige Resultat. Aber da in der dritten Gruppe die Geraden $a_{3} b_{1} c_{3}$ und $a_{3} b_{2} c_{4}$ durch zwei Paare conjugirt imaginärer Punkte $b_{1} b_{2}$ und $c_{3} c_{t}$ gehen, so sind sie conjugirt imaginäre Geraden, und folglich ist ihr Durchschnittspunkt $a_{3}$ reell. Dann versteht sich von selbst, dass auch $a_{4}$ reell sein muss, was überdies die vierte Gruppe bestätigt.

3. Aus diesen beiden Sätzen folgt unmittelbar: Wenn bei einer Curve dritter Ordnung aus einem Curvenpunkte zwei reelle und zwei imaginäre Tangenten an die Curve gelegt werden können, so hat jeder Curvenpunkt diese Eigenschaft.

4. Wenn von einem Curvenpunkte vier reelle Tangenten ausgehen, so besitzt die Curve allemal auch solche Punkte, bei denen die vier von ihnen ausgehenden Tangenten alle imaginär sind; und umgekehrt.

Beweis. Man kann, wie an einem anderen Orte *) gezeigt wurde, eine Curve dritter Ordnung stets als den geometrischen Ort der Durchschnitte entsprechender Strahlenpaare zweier projectivischer Strahleninvolutionen betrachten, und zwar genügt es dazu, zwei der Curve in einem der drei Systeme angehörige conjugirte Pole $o$ und $o^{\prime}$ als Scheitel der Involutionen zu nehmen, deren Strahlenpaare nach den Polepaaren desselben Systemes gehen. Alsdann sind die den Doppelstrahlen der einen Involution [o] entsprechenden Strahlenpaare der anderen $\left[o^{\prime}\right]$, da diese die Doppelstrahlen in je zwei zusammenfallenden Punkten schneiden, die aus $o^{\prime}$ an die Curve gehenden Tangenten. Nun darf man allerdings nicht schliessen, dass wenn die Doppelstrahlen in [o] reell sind, auch die Tangenten aus $o^{\prime}$ reell seien; denn es kann sehr wohl vorkommen und kommt in einem ausgedehnten Falle wirklich vor, dass einem reellen Doppelstrahle ein imaginäres Strahlenpaar entspricht, welches dann ein imaginäres Tangentenpaar bildet. Allein, wenn die Doppelstrahlen in [o] imaginär sind, so müssen auch die Tangenten aus $o^{\prime}$ imaginär sein; denn, da die Durchschnitte eines Doppelstrahles mit dem ihm entsprechenden Strahlenpaare die Berührungspunkte des letzteren sind, so würden, wenn einem imaginären Doppelstrahle ein reelles Strahlenpaar entspräche, zwei reelle Tangenten entstehen, deren Berührungspunkte imaginär sind, was nicht möglich ist. Sei nun $o$ ein Punkt der Curve, von welchem vier reelle Tangenten $o(a b c d)$ an die Curve gehen. Dann sind $a b, c d ; a c, b d ; a d, b c$ conjugirte Polepaare

*) Ueber die Curve dritter Ordnung, welche den geometrischen Ort der Brennpunkte einer Kegelschnittschaar bildet. Math. Annalen, Bd. 5, pag. 83. 
in den drei Systemen, und die Durchschnitte

$$
(a b, c d)=o^{\prime}, \quad(a c, b d)=o^{\prime \prime}, \quad(a d, b c)=o^{\prime \prime \prime}
$$

die dem $o$ in den drei Systemen conjugirten Pole. Jeden dieser drei Punkte kann man daher als den Scheitel einer Involution wählen, welche mit der Involution [o] zusammen die Curve erzeugt, und je nachdem man $o^{\prime}$ oder $o^{\prime \prime}$ oder $o^{\prime \prime \prime}$ wählt, bilden $o(a b, c d)$ oder $o(a c, b d)$ oder $o(a d, b c)$ conjugirte Strahlenpaare in [o]. Unter diesen Anordnungen aber giebt es immer eine, bei welcher die Strahlenpaare einander trennen, nämlich, wenn man die Strahlen $o(a b c d)$ so bezeichnet, dass sie in dieser Reihe auf einander folgen, die Paare $o(a c, b d)$. Bei dieser Involution sind also die Doppelstrahlen. imaginär, und folglich sind die aus dem zugehörigen conjugirten Pole $o^{\prime \prime}$ an die Curve gehenden Tangenten alle vier imaginär. - Dass umgekehrt eine Curve, welche Punkte mit vier imaginären Tangenten besitzt, auch Punkte mit vier reellen Tangenten haben muss, ist ohne Weiteres klar, da jedem Curvenpunkte ein Tangentialpunkt zugehört, von dem eine reelle, und daher nach dem zweiten Satze vier reelle Tangenten ausgehen.

Nach diesen Sälzen können alle Curven dritter Ordnung ohne Doppelpunkt in zwei Gattungen getheilt werden:

Zur ersten Gattung rechnen wir diejenigen Curven, aus deren Punliten theils vier reelle, theils vier imaginäre, niemals aber zwei reelle und zwei imaginäre Tangenten an die Curve gelegt werden können.

Die zweite Gattung bilden diejenigen Curven, bei denen aus jedem Punkte zwei reelle und zwei imaginäre Tangenten an die Curve gehen.

Diese beiden Curvengattungen haben nun aber auch wesentlich von einander verschiedene Formen. Bei der Betrachtung der Gestalten, welche die Curven dritter Ordnung darbieten, bemerke man, dass stets je zwei in's Unendliche sich erstreckende Curvenäste der nämlichen (geradlinigen oder parabolischen) Asymptote sich anschliessen. Solche zwei Aeste kann man dann als im Unendlichen zusammenhängend betrachten; denn lässt man eine Gerade sich um einen beliebigen festen Punkt drehen und sieht einen Curvenast als den Weg an, den ein Durchschnittspunkt dieses Astes mit der Geraden bei der Drehung der letzteren durchläuft, so setzt diese ihre Drehung continuirlich fort, wenn der Schnittpunkt den unendlich fernen Punkt des Astes erreicht, d. h. wenn die Gerade der betreffenden Asymptote (oder bei einer parabolischen Asymptote der Axe der Parabel) parallel wird, und der derselben Asymptote sich anschliessende zweite Curvenast ist dann als die Fortsetzung des Weges für den 
Schnittpunkt zu betrachten. Möbius *) sagt mit Recht, dass, ,die Eigenschaften welche einer ebenen Linie in Bezug auf ihre unendlichen Aeste zukommen, nicht zu den wesentlicheren gerechnet werden können"6. Aus diesem Grunde betrachtet er die Projection der Curve aus dem Mittelpunkte einer Kugel auf die Oberfläche derselben, weil man dadurch die Curve „,von ihren unwesentlicheren Eigenschaften entkleidet"6, und fügt hinzu: „,Je nachdem man die sphärische Curve bald auf diese, bald auf jene Ebene zurückprojicirt, werden bald diese, bald jene Theile der Curve sich in der Ebene als unendliche Aeste abbilden."6 Demgemäss werden wir solche Curvenstücke, deren unendliche Aeste paarweise derselben Asymptote sich anschliessen, als zusammenhängende Curvenstücke betrachten.

Dies vorausgeschickt folgt nun leicht, dass jede der ersten Gattung angehörige Curve, welche also sowohl Tangentialpunkte mit lauter reellen, als auch solche mit lauter imaginären Tangenten besitzt, nothwendig aus zwei ganz von einander getrennten (auch nicht im Unendlichen zusammenhängenden) Theilen bestehen muss. Denn ein continuirlicher Uebergang von reellen $\mathbf{z u}$ imaginären Tangenten kann nur dadurch eintreten, dass an der Uebergangsstelle zwei Tangenten zusammenfallen; das aber kann bei einer Curve dritter Ordnung ohne Doppelpunkt niemals vorkommen. Daher muss eine Curve der ersten Gattung immer aus zwei getrennten Theilen bestehen, der Art, dass der eine Theil lauter Punkte enthält, von denen vier reelle, der andere lauter solche, von denen vier imaginäre Tangenten ausgehen. Dass eine Curve dritter Ordnung nicht mehr als zwei von einander getrennte Theile besitzen kann, bedarf wohl keiner näheren Erörterung.

Es gilt aber auch das Umgekehrte. Wenn nämlich eine Curve dritter Ordnung ohne Doppelpunkt aus zwei getrennten Theilen besteht, so muss der eine Theil der einen, der andere den beiden anderen Asymptoten sich anschliessen, wenn daher imaginäre oder im Unendlichen zusammenfallende Asymptoten vorhanden sind, so müssen diese dem zweiten Theile angehören. Demnach erstreckt der erstere Theil sich in's Unendliche und nähert sich mit seinen beiden Aesten derselben Asymptote (wir wollen ihn den Theil $U$ nennen), während der andere (er möge $S$ heissen) entweder ein geschlossenes Oval bildet, oder aus zwei im Unendlichen zusammenhängenden Stücken besteht, deren unendliche Aeste paarweise den beiden anderen Asymptoten nach

*) Ueber die Grundformen der Linien der dritten Ordnung, pag. 8. 
Art einer Hyperbel sich anschliessen. (Oder es kann auch der Theil $S$ nur aus einem in's Unendliche gehenden Stücke bestehen, welcher die unendlich ferne Gerade berührt.) Lässt man nun eine Gerade sich parallel mit der stets reellen, dem Theile $U$ angehörigen, Asymptote verschieben, so trifft sie in jeder ihrer Lagen entweder den Theil $U$ oder $S$ oder keinen von beiden in zwei reellen Punkten. Bei dem Eintritte in und dem Austritte aus jedem dieser beiden Theile wird sie daher zur Tangente und liefert vier von dem unendlich fernen Punkte des Theiles $U$ ausgehende reelle Tangenten. Mithin gehört eine solche Curve allemal der ersten Gattung an, und man sieht zugleich, dass der Theil $U$ diejenigen Punkte enthält, von denen vier reelle Tangenten, der Theil $S$ also diejenigen, von denen vier imaginäre Tangenten ausgehen.

Hieraus folgt nun weiter, dass eine der zweiten Gattung angehörige Curve, bei welcher allen Punkten zwei reelle und zwei imaginäre Tangenten zugehören, immer aus einem einzigen vollständig zusammenhängenden Theile besteht.

Dies sind die Formunterschiede, die man mit Möbius als die wesentlichen bezeichnen kann. Sie bieten zugleich in der Beschaffenheit der von ihren Punkten ausgehenden Tangenten einen charakteristischen geometrischen Unterschied dar. Ausserdem kann nun jede der beiden erwähnten Gattungen nach demselben Princip in Unterabtheilungen gebracht werden, nach welchem man die Kegelschnitte einzutheilen pflegt; je nachdem nämlich die unendlich ferne Gerade ausser dem einen reellen Punkte, den sie stets mit der Curve gemein hat, diese noch in zwei imaginären, oder zwei reellen, oder zwei zusammenfallenden Punkten trifft, in welchen drei Fällen die Curve eine, oder drei geradlinige Asymptoten, oder endlich eine gerade und eine parabolische Asymptote hat. Demgemäss lassen sich alle Curven dritter Ordnung ohne Doppelpunkt in sechs Arten theilen, von denen zwei als Uebergangsfälle auftreten:

Erste Gattung. Die Curve besteht aus zwei getrennten Theilen $U$ und $S$; aus jedem Punkte von $U$ gehen vier reelle, aus jedem Punkte von $S$ vier imaginäre Tangenten an die Curve. $U$ erstreckt sich in's Unendliche und schliesst sich mit seinen beiden Aesten derselben Asymptote an.

a) Eine gerade Asymptote. $S$ bildet ein Oval.

b) Drei gerade Asymptoten. $S$ besteht aus zwei im Unendlichen zusammenhängenden Stücken, deren Aeste sich paarweise den beiden anderen Asymptoten nach Art einer Hyperbel anschliessen. 
c) Eine gerade und eine parabolische Asymptote (Uebergangsfall). $S$ besteht aus einem ins Unendliche gehenden Stücke, deren Aeste sich einer Parabel anschliessen (die unendlich ferne Gerade berühren).

Zweite Gattung. Die Curve besteht aus einem einzigen im Unendlichen zusammenhängenden Theile. Aus jedem Curvenpunkte gehen zwei reelle und zwei imaginäre Tangenten an die Curve.

a) Eine gerade Asymptote. Die Curve besteht aus einem Stŭcke, dessen unendliche Aeste sich der Asymptote anschliessen.

b) Drei gerade Asymptoten. Die Curve besteht aus drei in's Unendliche gehenden Stücken; je zwei nicht demselben Stücke angehörende Aeste schliessen sich derselben Asymptote an.

c) Eine gerade und eine parabolische Asymptote (Uebergangsfall). Die Curve besteht aus zwei ins Unendliche gehenden Stücken. Der eine Ast jedes Stückes schliesst sich der geraden, der andere der parabolischen Asymptote an.

II.

Die beiden im Vorigen von einander unterschiedenen Gattungen der allgemeinen Curven dritter Ordnung stehen zu einander in einer eigenthümlichen Beziehung, welche hervortritt, wenn man bei den Curven der ersten Gattung denjenigen Theil, welcher oben mit $U$ bezeichnet wurde, und der diejenigen Punkte enthält, von denen vier reelle Tangenten ausgehen, für sich allein betrachtet. Es wird sich zeigen, dass dieser Theil bemerkenswerthe Eigenschaften mit den Curven der zweiten Gattung gemeinschaftlich hat, und nicht bloss mit diesen, sondern auch mit denjenigen Curven dritter Ordnung, die einen isolirten Doppelpunkt besitzen.

Da nämlich von dem Theile $S$ der Curve erster Classe keine reellen Tangenten ausgehen, so enthält $U$ sämmtliche (reelle) Tangentialpunkte, $S$ keine. Daher liegen auf $U$ auch stets die Durchschnitte der reellen Asymptoten und die drei reellen Wendepunkte. Ferner aber lässt sich zeigen, dass, gerade wie bei den Curven der zweiten Gattung, von jedem auf $U$ liegenden Punkte immer zwei und nur zwei reelle Tangenten an $U$ gelegt werden können. Um dies einzusehen, sei $o$ irgend ein auf $U$ liegender Punkt, und $o(a b c d)$ die von ihm ausgehenden Tangenten, welche wir wieder so be- 
zeichnet annehmen wollen, dass diese Strahlen in der angegebenen Reihe auf einander folgen. Betrachtet man nun das von den Berührungspunkten $a b c d$ gebildete vollständige Viereck und dessen Diagonalpunkte

$$
o^{\prime}=(a b, c d), \quad o^{\prime \prime}=(a c, b d), \quad o^{\prime \prime \prime}=(a d, b c),
$$

welche mit $o$ zusammen ein neues Punktquadrupel bilden, so haben wir oben gesehen, dass die von $o^{\prime \prime}$ ausgehenden Tangenten alle vier imaginär sind, sodass $o^{\prime \prime}$ auf $S$ liegt. Da aber $S$ einen für sich geschlossenen Theil bildet, der entweder in einem endlichen Raume enthalten ist, oder sich nach Art einer Hyperbel oder Parabel in's Unendliche erstreckt, so hat jede reelle Gerade, welche $S$ in einem reellen Punkte trifft, mit diesem Theile noch einen reellen Punkt gemein. Nun wird $S$ in $o^{\prime \prime}$ von den Geraden $a c$ und $b d$ getroffen, mithin liegt von jedem dieser beiden Punktepaare der eine Punkt auf $S$, und also der andere auf $U$. Von den vier von einem auf $U$ liegenden Punkte ausgehenden reellen Tangenten berühren demnach allemal zwei den Theil $S$ und zwei den Theil $U$.

Nehmen wir, um etwas Bestimmtes zu haben, an, die Berührungspunkte $a b$ liegen auf $U$, cd auf $S$, so folgt nun weiter, da $a b$, $c d$ sich in $o^{\prime}, a d$, $b c$ aber in $o^{\prime \prime \prime}$ schneiden, dass $o^{\prime \prime \prime}$ ebenso wie $o^{\prime \prime}$ auf $S$, dagegen $o^{\prime}$ auf $U$ liegt. Da nun $o^{\prime}, o^{\prime \prime}, o^{\prime \prime \prime}$ die zu $o$ in den drei Systemen conjugirten Pole sind, so folgt: Bei den Curven der ersten Gattung ist die Vertheilung der conjugirten Pole der Art, dass diese in einem der drei Systeme (oo', $\left.o^{\prime \prime} o^{\prime \prime \prime}\right)$ stets auf demselben Theile der Curve liegen, in den beiden anderen Systemen (oo $\left.o^{\prime \prime}, o^{\prime} o^{\prime \prime \prime}\right)$ und $\left(o^{\prime \prime \prime}, o^{\prime} o^{\prime \prime}\right)$ dagegen stets auf verschiedenen Theilen. Bei den Curven der zweiten Gattung giebt es natürlich nur ein System reeller conjugirter Pole, das dem vorhin zuerst genannten analog ist.

Der auf $U$ liegende Punkt $o^{\prime}$ liegt mit den beiden auf demselben Theile befindlichen Punkten $a, b$ in gerader Linie, daher hat man: Zieht man von einem auf $U$ liegenden Punkte die beiden Tangenten an diesen Theil mit den Berúhrungspunkten $o, o^{\prime}$, und von dem einen, $o$, auf's Neue die Tangenten an den nämlichen Theil mit den Berïhrungspunkten $a, b$, so liegt der andere, $o^{\prime}$, mit den beiden letzteren $a, b$ in gerader Linie. Und dieser Satz behält seine Geltung, wenn statt des Theiles $U$ einer Curve erster Gattung, eine aus der zweiten Gattung substituirt wird.

Im Folgenden soll ausschliesslich nur von reellen Punkten die Rede sein, daher werden wir den Zusatz „reell“ als selbstverständlich in der Folge weg- 
lassen. Ausserdem gelten die folgenden Betrachlungen ebensowohl von dem Theile $U$ einer Curve der ersten Gattung, wie von einer Curve der zweiten Gattung, es mögen daher beide unter der Benennung „eine Curve $U^{\text {" zu- }}$ sammengefasst werden.

Die Durchschnitle der harmonischen Polare eines Wendepunktes mit einer Curve dritter Ordnung sind die Berührungspunkte der von dem Wendepunkte ausgehenden Tangenten, während die vierte Tangente die Wendetangente ist; daher wird eine Curve $U$ von einer harmonischen Polare stets nur in einem Punkle geschnitten. Sind nun $d, d^{\prime}, d^{\prime \prime}$ diese Durchschnitte, den Wendepunkten $\omega, \omega^{\prime}, \omega^{\prime \prime}$ resp. zugehörig, so sind sie zugleich die den letzteren conjugirten Pole in demselben Systeme. Da aber $\omega, \omega^{\prime}, \omega^{\prime \prime}$ in einer Geraden liegen, so bilden diese mit $d, d^{\prime}, d^{\prime \prime}$ die sechs Ecken eines vollständigen Vierseits, d. h. $\omega d^{\prime} d^{\prime \prime}, \omega^{\prime} d^{\prime \prime} d, \omega^{\prime \prime} d d^{\prime}$ liegen in je einer Geraden, oder: Die Durchschnitte einer Curve $U$ mit den harmonischen Polaren zweier Wendepunkte liegen mit dem dritten Wendepunkte in gerader Linie.

Für das Folgende muss zuerst nachstehender Satz bewiesen werden : Lässt man den Berïhrungspunkt einer Tangente eine Curve $U$ durchlaufen, so durch$l_{\ddot{a} u f t}$ der zugehörige Tangentialpunkt diese Curve in entgegengesetzter Richtung.

Beweis. So lange der Berührungspunkt seine Bewegungsrichtung nicht ändert, behält auch der Tangentialpunkt die seinige im Allgemeinen bei und könnte sie höchstens beim Ueberschreiten eines Wendepunktes ändern, weil nur an diesen Stellen die Tangente den Sinn ihrer Drehung umkehrt. Zieht man aber in der Nähe eines Wendepunktes eine Tangente an die Curve, so muss der Tangentialpunkt auf der entgegengesetzten Seite des Wendepunktes liegen. Denn die Curve kehrt in dem Berührungspunkte der Tangente ihre convexe Seite zu und kann derselben nicht eher wieder begegnen, bis die Convexität in eine Concavität übergegangen ist, sodass zwischen dem Berührungspunkte und dem Tangentialpunkte mindestens ein Wendepunkt enthalten sein muss. Da nun diese beiden Punkte in dem Wendepunkte zusammenfallen, so nähern sie sich dem letzteren von entgegengesetzten Seiten, überschreiten ihn und gehen dann wieder nach entgegengesetzten Seiten auseinander. Hiernach ändert also ein Tangentialpunkt beim Ueberschreiten eines Wendepunktes seine Bewegungsrichtung nicht, ,wenn der Berührungspunkt dies nicht thut, und da der erstere an diesen Stellen sich in entgegengesetzter Richtung bewegt, wie der letztere, so findet dasselbe durch die ganze Curve hindurch statt. 
Hieraus folgt sofort, dass die Berührungspunkle $d, d^{\prime}, d^{\prime \prime}$ der aus den Wendepunkten $\omega, \omega^{\prime}, \omega^{\prime \prime}$ an eine Curve $U$ gezogenen Tangenten zwischen den Wendepunkten enthalten sein müssen, oder, was dasselbe ist, diese zwischen jenen. (Wir stellen uns dabei immer die Curve als im Unendlichen zusammenhängend vor.) Um dies zu zeigen, sei $t$ ein Tangentialpunkt, $b$ und $\beta$ die beiden ihm zugehörigen Berührungspunkie. Jedesmal wenn einer der letzteren in einen Punkt $d$ fällt, fällt der andere und gleichzeitig auch $t$ in den zugehörigen Wendepunkt $\omega$. Nehmen wir an, $t$ und $b$ befinden sich in $\omega$, also $\beta$ in $d$. Lässt man nun diese Punkte sich bewegen, so schlagen nach dem vorigen Satze $b$ und $\beta$ die nämliche, $t$ aber die entgegengesetzte Richtung ein. Lässt man $b$ von $\omega$ nach $d$, also gleichzeitig $\beta$ von $d$ nach $\omega$ (in derselben Richtung, also auf der anderen Seite der Curve) gehen, so bewegt sich $t$ in entgegengesetzter Richtung um die ganze Curve herum von $\omega$ nach diesem Punkte zurück. Dabei muss also $t$ sowohl dem $b$, als auch dem $\beta$ begegnen, und da dies nur in einem Wendepunkte geschehen kann, so liegt ein Wendepunkt $\omega^{\prime}$ zwischen $\omega$ und $d$, und der dritte $\omega^{\prime \prime}$ zwischen $d$ und $\omega$. (Man denke sich diese Punkte etwa symbolisch auf der Peripherie eines Kreises vertheilt.) Wir nehmen nun ferner $\omega^{\prime}$ als Ausgangspunkt für $t$ und $b$, sodass dann $\beta$ von $d^{\prime}$ ausgeht, und lassen $t$ von $\omega^{\prime}$ über $d$ nach $\omega^{\prime \prime}$ sich bewegen, also ohne einen Wendepunkt zu überschreiten; dann geht $b$ in entgegengesetzter Richtung von $\omega^{\prime}$ nach $d^{\prime \prime}$, ohne seinerseits einen Wendepunkt oder einen anderen Punkt $d$ zu überschreiten; $\beta$ aber geht in der der vorigen gleichen Richtung von $d^{\prime}$ nach $\omega^{\prime \prime}$, ebenfalls ohne einen Wendepunkt oder einen anderen Punkt $d$ zu treffen. Mithin liegt $d^{\prime \prime}$ zwischen $\omega^{\prime}$ und $\omega$, und $d^{\prime}$ zwischen $\omega$ und $\omega^{\prime \prime}$. Demnach ist die Reihenfolge dieser Punkte auf der Curve die folgende:

$$
d \omega^{\prime} d^{\prime \prime} \omega d^{\prime} \omega^{\prime \prime} d,
$$

und man sieht, dass jeder Wendepunkt zwischen denjenigen zwei Punkten d enthalten ist, mit denen er in gerader Linie liegt.

Fasst man nun die so durch die Punkte $d$ auf der Curve $U$ entstehenden Abschnitte in's Auge, in denen jedes Mal nur ein einziger Wendepunkt enthalten ist, so erkennt man leicht, dass, wenn man aus einem Punkte $\boldsymbol{t}$ die beiden Tangenten an die Curve $U$ zieht, immer ein Berührungspunkt und mur einer in demselben Abschnitte liegt, wie der Tangentialpunkt $t$. Denn während $t$ z. B. die Strecke $\omega \omega^{\prime}$ durchläuft, die den Punkt $d^{\prime \prime}$ enthält, beschreibt der eine Berührungspunkt $b$ die Strecke $\omega d^{\prime}$, der andere $\beta$ die 
Strecke $d \omega^{\prime}$ So lange also $t$ zwischen $\omega$ und $d^{\prime \prime}$ bleibt, liegt $b$ mit ihm in demselben Abschnitte, nämlich in $d^{\prime} d^{\prime \prime}$; gelangt aber $t$ in die Strecke $d^{\prime \prime} \omega^{\prime}$, so liegt der andere Berührungspunkt $\beta$ mit ihm in demselben Abschnitte, nämlich in $d^{\prime \prime} d$.

Zieht man jetzt aus einem Punkte $b_{0}$, der etwa in dem Abschnitte $d^{\prime} d^{\prime \prime}$ liege, welche den Wendepunkt $\omega$ enthält, diejenige Tangente an die Curve $U$, deren Berührungspunkt $b_{1}$ in denselben Abschnitt fällt, sodann aus $b_{1}$ wieder diese Tangente mit dem Berührungspunkte ' $b_{2}$, und aus $b_{2}$ die entsprechende Tangente $b_{2} b_{3}$, so liegen die Punkte $b_{1}, b_{2}$ auf der einen, $b_{1}, b_{3}$ auf der anderen Seite des Wendepunktes $\omega$. Offenbar können weder $b_{0} b_{2}$ noch $b_{1} b_{3}$ zusammenfallen, sonst erhielte man eine Doppeltangente, also muss entweder $b_{0}$ oder $b_{2}$ näher an dem Wendepunkte liegen, als der andere dieser beiden Punkte. Schreitet man nun von $b_{0}$ über $\omega$ nach $b_{1}$ vorwärts und geht in derselben Richtung um die ganze Curve nach $b_{2}$ weiter (wobei man. ein oder mehrere Male durch das Unendliche hindurch muss), so wird dabei $b_{0}$ überschritten oder nicht überschritten, je nachdem $b_{2}$ oder $b_{0}$ der näher an $\omega$ gelegene Punkt ist. Dann bewegt sich gleichzeitig der dem Tangentialpunkte zugehörige Berührungspunkt in der entgegengesetzten Richtung von $b_{1}$ über $\omega$ und $b_{2}$ nach $b_{3}$, und, um nach $b_{3}$ zu gelangen, muss $b_{1}$ überschritten werden oder nicht, je nachdem vorhin $b_{0}$ überschritten wurde oder nicht. Wenn daher $b_{2}$ näher (oder weiter) an $\omega$ liegt als $b_{0}$, so liegt auch $b_{3}$ näher (oder weiter) als $b_{1}$. Man sieht also, dass der Berührungspunkt bei dem fortgesetzten Tangentenziehen entweder sich unaufhörlich dem Wendepunkte nähert, oder sich unaufhörlich von diesem entfernt. Nun kann aber der letztere Fall nicht stattinden. Denn, da dabei von der Annäherung an eine Grenzlage nicht die Rede sein kann, indem bis zu dem nächsten Wendepunkte kein Punkt auf der Curve sich vorfindet, in welchem eine solche eintreten könnte, so würde, wenn der Berührungspunkt sich unaufhörlich von dem Wendepunkte entfernte, einmal eine Lage eintreten, bei welcher ein Berührungspunkt noch innerhalb des in Betracht kommenden Abschnittes $d^{\prime} d^{\prime \prime}$ läge, der nächste aber schon ausserhalb desselben fiele, sodass es dann nicht möglich wäre, eine Tangente zu ziehen, deren Berührungspunkt mit dem Tangentialpunkte in dem nämlichen Abschnitte enthalten ist, was gegen einen früheren Satz streitet. Der nämliche Widerspruch würde sich ergeben, wenn ein Berührungspunkt gerade z. B. auf $d^{\prime \prime}$ fiele, denn dann liegt, wie früher 
gezeigt wurde, der nächste noch innerhalb der Strecke $\omega d^{\prime}$, der darauf folgende aber müsste über $d^{\prime \prime}$ hinaus fallen. Demnach nähert sich bei diesem fortgesetzten Tangentenziehen der Berïhrungspunkt unaufhörlich dem in dem betreffenden Abschnitte liegenden Wendepunkte, indem er abwechselnd von der einen Seite des letzteren auf die andere hinübergeht.

Die vorstehenden Sätze gelten ebensowohl von den Curven der zweiten Gattung, als von dem Theile $U$ der Curven erster Gattung. Sie sind aber an einem anderen Orte ${ }^{*}$ ) auch von den speciellen Curven dritter Ordnung bewiesen worden, welche einen isolirten Doppelpunkt besitzen. An derselben Stelle wurden von diesen Curven noch andere Eigenschaften mitgetheilt, und namentlich gewisse Vielecke näher betrachtet, welche der Curve gleichzeitig ein- und umgeschrieben sind, und dadurch entstehen, dass man die successiven Tangentialpunkte eines Berührungspunktes verfolgt und die Fälle untersucht, in denen ein späterer Tangentialpunkt mit dem ersten Berührungspunkte zusammenfällt. Auch diese Eigenschaften lassen sich bei den allgemeinen Curven $U$ wiederfinden, doch wollen wir auf dieselben hier nicht näher eingehen, da sie besser unter einem anderen Gesichtspunkte betrachtet werden.

Aus der Uebereinstimmung, welche die betrachteten Curven in ihren Eigenschaften zeigen, lässt sich ersehen, auf welche Weise eine Curve der ersten Gattung in eine der zweiten Gattung übergeht. Indem nämlich bei einer Curve der ersten Gattung der Theil $S$ in einen Punkt zusammenschrumpft, entsteht eine Curve mit einem isolirten Doppelpunkt, und diese geht in eine Curve der zweiten Gatlung über, dadurch dass der Theil $S$ in allen seinen Punkten imaginär wird. Bei diesen Uebergängen behält aber der Theil $U$ die Eigenschaften, die ihm, wenn er für sich allein betrachtet wird, in seinen reellen Punkten zukommen, bei.

Die Curven dritter Ordnung mit einem eigentlichen Doppelpunkte verhalten sich anders. Eine solche entsteht aus einer Curve der ersten Gattung, indem der Theil $S$ sich mit dem Theile $U$ verbindet und zur Schleife wird, denn von dieser gehen keine reellen Tangenten an die Curve. Dabei fallen zwei der auf $U$ liegenden reellen Wendepunkte und die Punkte $d$ in den Doppelpunkt, ebenso auch fünf der auf $S$ liegenden reellen Punkte $d$, sodass

*) Ueber fortgesetztes Tangentenziehen an Curven dritter Ordnung nit einem Doppel- oder Rtickkehrpunkte. Math. Annalen Bd. 1. 
von den neun reellen Durchschnitten der Curve mit den drei reellen harmonischen Polaren nur ein einziger auf der Schleife liegender übrig bleibt, alle übrigen aber sich in dem Doppelpunkte vereinigen. Diese Curven gehören weder zu der ersten, noch zu der zweiten Gattung, oder wenn man will, gleichzeitig zu beiden, indem sie mit jener das gemein haben, dass von ihren Punkten zum Theil nur reelle, zum Theil nur imaginäre Tangenten ausgehen, mit dieser dagegen das, dass sie nur aus einem einzigen zusammenhängenden Theile bestehen. Der Uebergang von reellen zu imaginären Tangenten wird bei ihnen durch das Zusammenfallen derselben im Doppelpunkte vermittelt.

Prag, 11. November 1871. 